\title{
Pseudorapidity spectra of secondary particles emitted in the relativistic nucleus-nucleus collisions
}

\author{
M. K. Suleymanov ${ }^{1}$ \\ COMSATS Institute of Information Technology \\ Park Road Shahzad Town, Islamabad, Pakistan \\ Veksler and Baldin Laboratory of High Energy Physics, JINR \\ Dubna 141980, Russia \\ E-mail: mais_suleymanovecomsats.edu.pk
}

\section{B. Z. Belashev}

Institute of Geology, Karelian Research Center, Russian Academy of Sciences St. Petrozavodsk, Russia

E-mail: belashev@krc.karelia.ru

\section{S. Vokal}

P. J. Šafárik University

Košice, Slovakia

E-mail: stanislav.vokal@upjs.sk

\section{A. S. Vodopianov}

Veksler and Baldin Laboratory of High Energy Physics, JINR

Dubna 141980, Russia

E-mail: vodopiandsunhe.jinr.ru

\section{J. Vrláková}

P. J. Šafárik University

Košice, Slovakia

E-mail: vrlakova@upjs.sk

\section{O. B. Abdinov}

H.M. Abdullayev Institute of Physics NAS

Baku, Azerbaijan

E-mail: abdinovaphysics.ab.az

\section{Ajaz}

COMSATS Institute of Information Technology Park Road Shahzad Town, Islamabad, Pakistan

Department of Physics, Abdul Wali Khan University

Mardan, Khyber PukhtoonKhwa, Pakistan

E-mail: Muhammad.Ajaz@cern.ch 


\section{Ali Zaman}

COMSATS Institute of Information Technology

Islamabad, Pakistan

E-mail: ali_zaman@comsats.edu.pk

\section{K. H. Khan}

COMSATS Institute of Information Technology

Islamabad, Pakistan

E-mail: kamal.khandcern.ch

\section{Sh. Khalilova}

H.M. Abdullayev Institute of Physics NAS

Baku, Azerbaijan

E-mail: shahla.ganbarova@cern.ch

We discuss new results connected with more detail structure of the pseudorapidity spectra of charged relativistic particles with $\beta>0.7$ produced in $\mathrm{Si}$ (at energy $4 \mathrm{~A} \mathrm{GeV}$ and $14 \mathrm{~A} \mathrm{GeV}$ ), Au (at energy 11.6 $\mathrm{A} \mathrm{GeV}$ ) and $\mathrm{Pb}+\mathrm{Em}$ (at energy $158 \mathrm{~A} \mathrm{GeV}$ ) reactions. The relativistic nucleus beams were obtained from AGS and SPS machines. The pseudorapidity spectra were analyzed using Maximum Entropy Methods. The number of fast target protons: g-particles are used to fix the centrality of collisions. Using the method we found some selected pseudorapidity values - nontrivial structure, the number of which depends on energy; increases from 2 to 4 with energy.

International Baldin Seminar on High Energy Physics Problems September 10-15, 2012

JINR, Dubna, Russia

\footnotetext{
${ }^{1}$ Spekaer
} 


\section{Introduction}

In high energy hadron-nuclear and nuclear-nuclear experiments some plateaus were observed visually in central area of pseudorapidity $(\eta)$. The existence of plateau is very important for theoretical estimation. For example, in paper [1] the space-time evolution of the hadronic matter produced in the central rapidity region in extreme nucleus-nucleus collisions has been described. It is found that quark-gluon plasma is produced at a temperature of the order of $200 \mathrm{MeV}$, which is in agreement with previous studies. The authors commented that the description relies on the existence of a flat central plateau and on the applicability of hydrodynamics. The experimentally observed plateau could be the sum of different contributions connected for example, to the fragments of various dynamics. In the framework of the thermalized cylinder model [2] it is a natural result of longitudinal extension. So the new results which could confirm the existence of the plateau are required.

In the paper [3] the methods of a posteriori increase the resolution of the spectral lines - Fourier transformation and maximum entropy methods were applied to obtain more detailed information on the structure of pseudorapidity spectra of relativistic particles emitted in the $\mathrm{Au}($ at $11.6 \mathrm{AGeV})$ and $\mathrm{Pb}($ at $158 \mathrm{AGeV})$ induced reactions at high energies. The central plateau and shoulder are seen visually on the spectra.

Both methods demonstrate some complex structure of the spectra. The maximum entropy method can confirm the existence of some selected pseudorapidity values $\left(\eta_{c}\right)$ which would be connected with the boundary values of the central plateau and the values of the pseudorapidity shoulder. The numbers of $\eta_{\mathrm{c}} \mathrm{s}$ were different for $A u+E m$ and $P b+E m$ reactions, for $P b+E m$ the number is greater than for the $A u+E m$. In the paper only two sets of experimental data were used, apparently it is not simple to make any conclusion on energy and the mass dependences for the results. The results coming from the methods were in form of some peaks which didn't fit to do any quantitative comparisons. Furthermore the authors didn't consider any simulation data coming from codes. These are some of the shortcomings of the results which are considered in this work.

2.we made wider the energy region of the reactions by including two new sets of experimental data: $\mathrm{Si}+\mathrm{Em}$ at 4.0 and $14.0 \mathrm{AGeV}$;

3. the peaks coming from the method were fitted to get quantitative results on the positions of the peaks $\left(\eta_{\mathrm{c}}\right)$, their widths $(\mathrm{W})$ and heights $(\mathrm{H})$;

4.the simulation data coming from the Modified Cascade model [4] were analyzed simultaneously with experimental data.

\section{On the method}

Pseudorapidity distributions were considered in the framework of the $s(i)=\sum_{i=1}^{M} h(i, j) f(j)+n(i)(i=1, \ldots M) \quad$ model, where $s$ is the estimation of the $z-$ distribution (smoothed distribution) of the $\eta$-spectra with the smoothed fluctuations, $h$ is the blurring function, $n$ is the additive noise, $f$ is the estimation of the distribution which defines the structure of $z$-distribution and $M$ is the number of steps in the histogram. Reconstruction of the structure for pseudorapidity distributions of secondary particles was done by a posteriori decreasing the widths of its components [5]. As a 
result of the artificial decreasing of the widths at constant positions, the resolution, the contrast and self-descriptiveness of estimations grow. The maximum entropy method (MEM) [6] was applied to decrease the width for the distribution components of the given values. Such data processing is especially evident for the case of Gaussian or Lorentz (Breit-Wigner) forms of components.

The MEM removes the given blurring function from distribution and maximizes the entropy of estimation at performance of models restrictions. The method could give the stable estimations at levels of additive noise $25 \%$. It works with narrow and wide, space-invariant and no space-invariant blurring functions [6]. The narrow blurring functions could detect profound details of structure, the wide ones leave in estimations of distributions wide intensive components. To analyze the $\eta$-distributions of the secondary particles and search for the existence of central plateau the MEM seems to be more preferable. The statistical fluctuations of the spectra were deleted using wavelettransformation of distribution [7] at the first stage of the processing. This is equivalent to the removal of high-frequency parts of signal from the spectrum. This procedure is effective at high level noise and differs considerably from the traditional averaging of histograms on counting and maintainsing in estimations the smoothed fluctuations. The algorithm was realized in package Wavelet Toolbox of the computer mathematics system MATLAB by means of a $w d c b m$ command, using approximating $c$ and detailing $l$ factors of wavelet decomposition of distribution and parameters alpha $=1: 02$ and $m=$ $l$ (1) [7]. To avoid occurrence of small (on absolute values) negative values in the estimation those smaller than 30 were attributed to the initial given histograms data. The obtained smoothed z-distribution of $\eta$-spectra were processed using the model / algorithm. In the next step the components widths were reduced assuming the forms of components were Gaussian function in the case of MEM. In the method the computing program MEMFR [8] and the blurring function $h(i ; j)=e^{[-(0.078(\eta(i)-\eta(j)) / \delta \eta) 2]}$ were taken in next analysis of the $\eta$-spectra. For the convenience of interpretation of the processing results the maximum from the estimations was fetched to the maximal value of the initial histogram.

Presentation of the pseudorapidity distributions for secondary particles as a superposition of peaks has allowed to estimate positions (P), amplitudes(A) and widths (S/G) of peaks using the genetic algorithm [9]. It was done with the command patternsearch [10] in MATLAB system. The algorithm gives the values of parameters which are global minimums function of least squares The errors of the parameters and the smoothed distributions were calculated on 30 values received at stochastic transformation of the histogram within the limits of three standard deviations.

\section{Used experimental data}

The stacks of NIKFI $B R$ - 2 nuclear emulsions were irradiated horizontally by $28 \mathrm{Si}$ beam at 4 [11] and $14 \mathrm{~A} \mathrm{GeV} \mathrm{[12],} \mathrm{by} 208 \mathrm{~Pb}$ beam at $158 \mathrm{~A} \mathrm{GeV/c}$ (Experiment EMU12 at the CERN SPS [13]) and by $197 \mathrm{Au}$ beam at $11.6 \mathrm{~A} \mathrm{GeV=c}$ (Experiment $E 863$ at $B N L A G S$ [14]). The projectile and target nuclei are disintegrated in the interactions 
of relativistic nuclei. According to the geometrical picture, there are in such collisions an interaction area and non-overlapping parts of both nuclei disintegrated to fragments due to the obtained excitation. As a result of an impact parameter variation, there appear events with the emission of secondaries of different types and energies within a wide range of multiplicity. The used emulsion method allows to measure multiplicities and angles of any charged particles and charge of projectile fragments. Secondary charged particles used in this study were classified into the (1) relativistic (shower) $s$-particles $\left(N_{s}\right)$, fast singly charged particles with $\beta>0.7$. This group includes particles produced in the interactions (mainly pions), relativistic singly charged projectile fragments as well as those singly charged particles knocked-out from the target nucleus. (2) fast target fragments, $g$-particles $\left(N_{g}\right)$, with $0.23 \leq \beta \leq 0.7$. They consist mainly of recoil protons from the target. In this work we have analyzed $1322 \mathrm{Si}+\mathrm{Em}$ (at $4 \mathrm{AGeV}$ ), $1093 \mathrm{Si}+\mathrm{Em}$ (at $14 \mathrm{AGeV}$ ), $628 \mathrm{~Pb}+\mathrm{Em}$ and $1185 \mathrm{Au}+\mathrm{Em}$ collisions found by the along the track scanning.

\section{Obtained results}

The main purpose of the investigations is to get information on structure of the pseudorapidity $(\eta=-\log (\tan (\theta / 2))$, where $\theta$ is polar angle in lab frame) spectra of single-charged relativistic particles with $\beta>0.7$ emitted in the $\mathrm{Si}, \mathrm{Au}$ and $\mathrm{Pb}$ induced reactions at energies 4.0-158.0 A GeV.

The Fig. 1 shows the experimental $\eta$-distribution for the $s$-particles produced in the reactions $\mathrm{Si}+\mathrm{Em}$ (4 and $14 \mathrm{AGeV}), \mathrm{Au}+\mathrm{Em}(11.6 \mathrm{AGeV})$ and in the reaction $\mathrm{Pb}+\mathrm{Em}(158 \mathrm{AGeV})$. The arrows point up some visually selected points for $\mathrm{Pb}+\mathrm{Em}$ interactions. The central plateau and shoulder are seen visually on the spectra.

It is not real thing to take the values of the $\eta_{c} s$ from the figure with adequate accuracy. So in paper [3] the methods of a posteriori increase the resolution of the spectral lines Fourier transformation and maximum entropy methods were applied to take the values for the $\eta_{c}$ from the spectra of relativistic particles emitted in the $A u(a t 11.6 \mathrm{AGeV})$ and $\mathrm{Pb}($ at $158 \mathrm{AGeV}$ ) induced reactions at high energies. Both methods demonstrate complex structure of the spectra. The maximum entropy method can confirm the existence of some $\eta_{c}$ which would be connected with the central plateau and the 


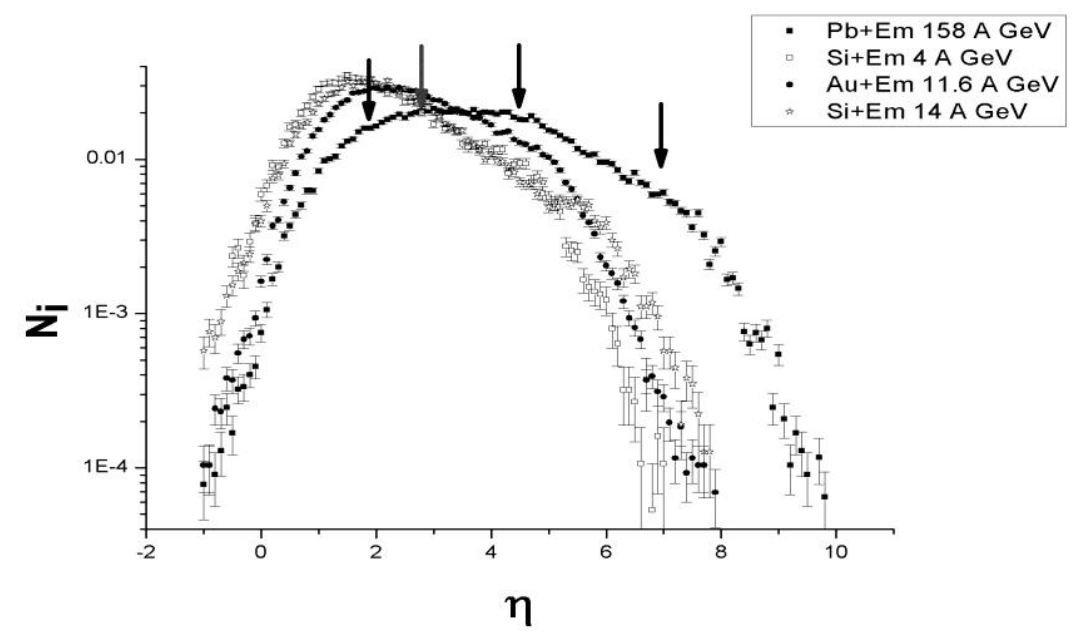

Fig.1. The $\eta$-distribution of single-charged relativistic particles with $\beta>0.7$ emitted in the $\mathrm{Si}, \mathrm{Au}$ and $\mathrm{Pb}$ induced reactions at energies 4.0-158.0 A GeV. The arrows point out the probable positions for selected values of the $\eta$.

shoulders. In paper [3] we presented results for $A u E m($ at $11.6 \mathrm{AGeV})$ and $\mathrm{PbEm}($ at 158 $A G e V$ ) without any competitions with result coming from the codes.

In this paper we made some enhancement in our investigation by adding the new experimental data on SiEm (at 4 and $14 \mathrm{AGeV}$ )-reactions. Only the MEM's results are considered because the method could give more clean results [3]. The results are fitted and compared with ones coming from the model [4] .

The Fig.2a-d and 3a-d demonstrate the results of the process, the data using the MEM (it was described in the section 2). We can say that visual observation shows that:

- the number of peaks, their positions (P), height (A) and widths (S) change as a function of energy and mass of the projectile;

- the method gives different results for the model and experimental data.

To characterize quantitatively the peaks the distributions from the Fig. 2 and 3 are fitted using the algorithm has been mentioned in the Section 2. The obtained values for the $\eta_{\mathrm{c}}$ are shown it Fig. 4a-d. The method could also define forth peaks in 3 cases. As we can see from Fig.4a-d the values for the $\eta_{\mathrm{c}}$ at:

- the first peaks don't depend on the beam energy and could be described completely by the model (Fig.4a);

- the second peaks depend on the beam energy in the region of $\mathrm{E}<10 \mathrm{AGeV}$ and could not be described completely by the model, in the region of $\mathrm{E}>10 \mathrm{AGeV}$ and don't depend on the energy (Fig.4b);

the third peaks could not be described completely by the model in the region of energy E $>10 \mathrm{AGeV}$ and don't depend on the energy (Fig.4c). 
As we have mentioned above the method could also define forth peaks in 3 cases (Fig.4d). We have analyzed the cases separately taking into account the number of gparticles. The Fig. 5 shows the fitting results for the cases which contain the fourth peaks: AuEm (at $11.6 \mathrm{AGeV}$ ) for all experimental events (Fig.5 top panel); $\mathrm{PbEm}$ (at $158 \mathrm{AGeV}$ ) for simulated events with a number of $\mathrm{g}$ particles $N_{g}>20$ (Fig.5 middle panel); $\mathrm{PbEm}$ (at $158 \mathrm{AGeV}$ ) for experimental events with a number of $\mathrm{g}$ particles $N_{g}=15-20$ (Fig.5 bottom panel). One can see that:

- in the first case amplitude of fourth peak (A4) is very small, i. e., 10 times less than that for the first peak (A1) and defined with error more than 50\%. The width of the peak also defined with error greater than $50 \%$ and that is why we ignored the peak;

- in the second case the first peak is not reasonable too due to the fact that in this case the position for the first peak (P1) could be defined with $100 \%$ error and the amplitude with error close to $50 \%$, it is really 3 peak case .

- in the last case : PbEm (at $158 \mathrm{AGeV}$ ) , $N_{g}=15-20$ all 4 peaks could be defined reasonably and could be considered as a 4 peak case only.

For the last case, the value of the $P 4=6.3 \pm 0.2$,-is in the stripping area of $\eta$. But the number of g-particles for this case is $N_{g}=15-20$ so it is not stripping particles. So we support that the case may be connected with some special dynamics other than dynamics for-previous 3 cases. We believe that in the last case we have some medium effect. To be sure we are going to continue the investigation using other models which could include medium effects too.
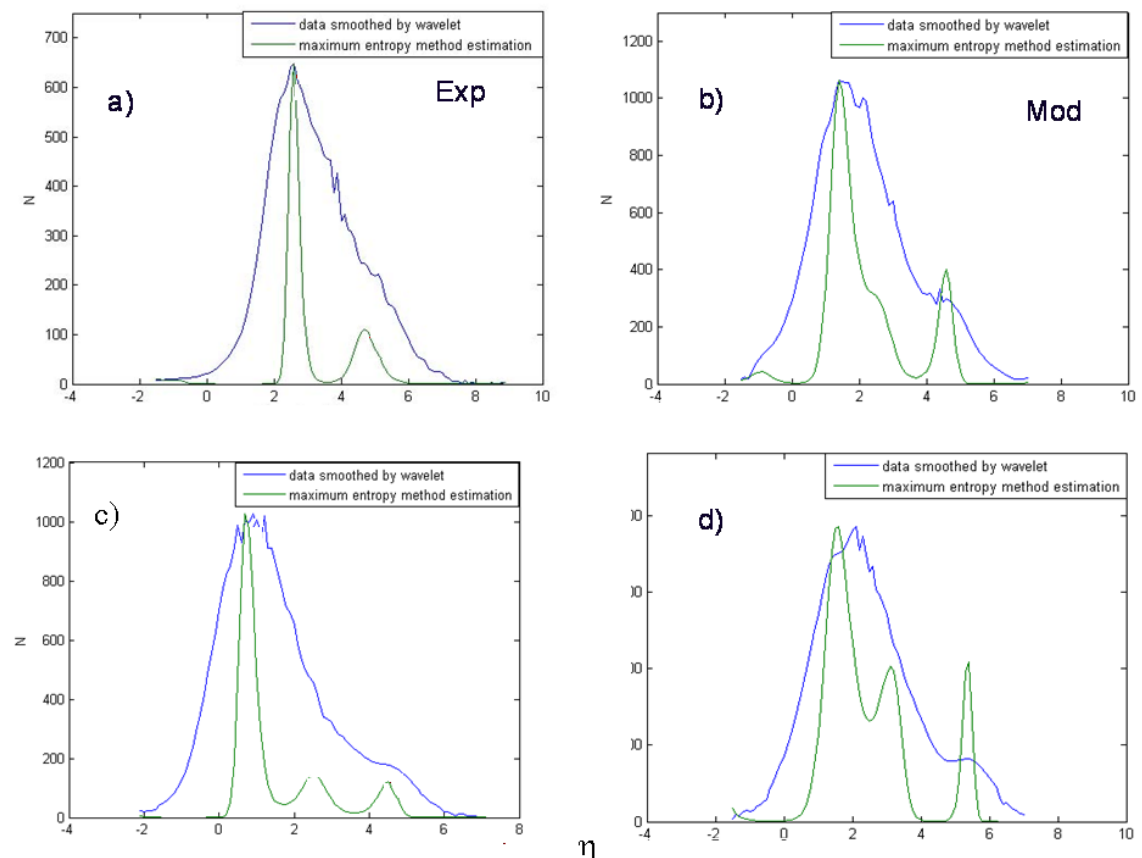

Fig. 2 a-d. MEM results (new figure) for the experimental (left panels) and model (right panels) data on s-particle $\eta$ distributions foe $\mathrm{Si}+\mathrm{Em}$-reactions at $4 \mathrm{AGeV}$ (top panels) and $14 \mathrm{AGeV}$ (bottom panels). 

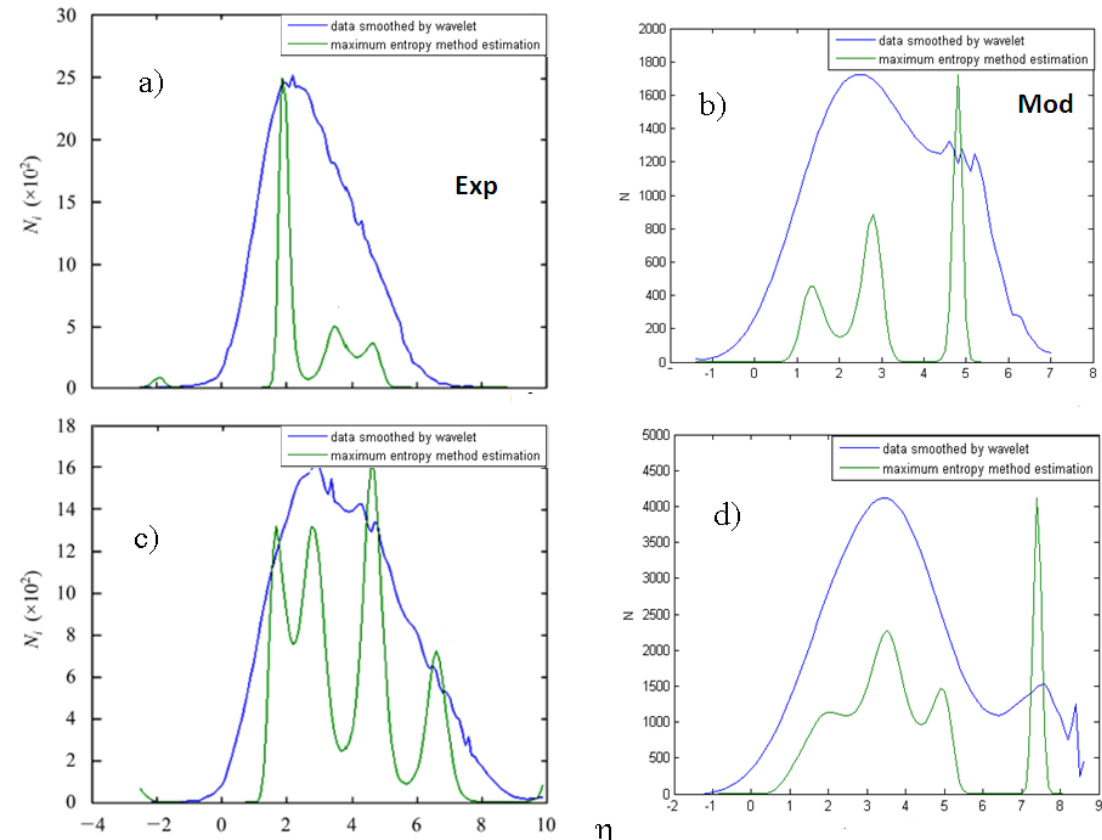

Fig.3 a-d. MEM results for the experimental (left panels are Figures from the paper [3]) and model (right panels new figures) data on s-particle $\eta$ distributions for $\mathrm{Au}+\mathrm{Em}$-reactions at $11.6 \mathrm{AGeV}$ (top panels) and $\mathrm{Pb}+\mathrm{Em}$ at $158 \mathrm{AGeV}$ (bottom panels).

\section{Conclusion.}

1. The methods of a posteriori increase the resolution of the spectral lines maximum entropy methods were applied to get more detail information on a structure of pseudorapidity spectra of relativistic particles emitted in the $\mathrm{Si}, \mathrm{Au}$ and $\mathrm{Pb}$ induced reactions at high energies. The spectra continue visually some selected values for the pseudorapidity.

2. The method could extract some selected values for $\eta$ too which are very close to observed visually ones.

3. The number of peaks increases with energy from 2 to 4 .

4. The method could extract some selected values of $\eta$ for simulated data coming from the Modified Cascade model too. Comparisons of the results coming from the experiment and the model show that the results are different. 


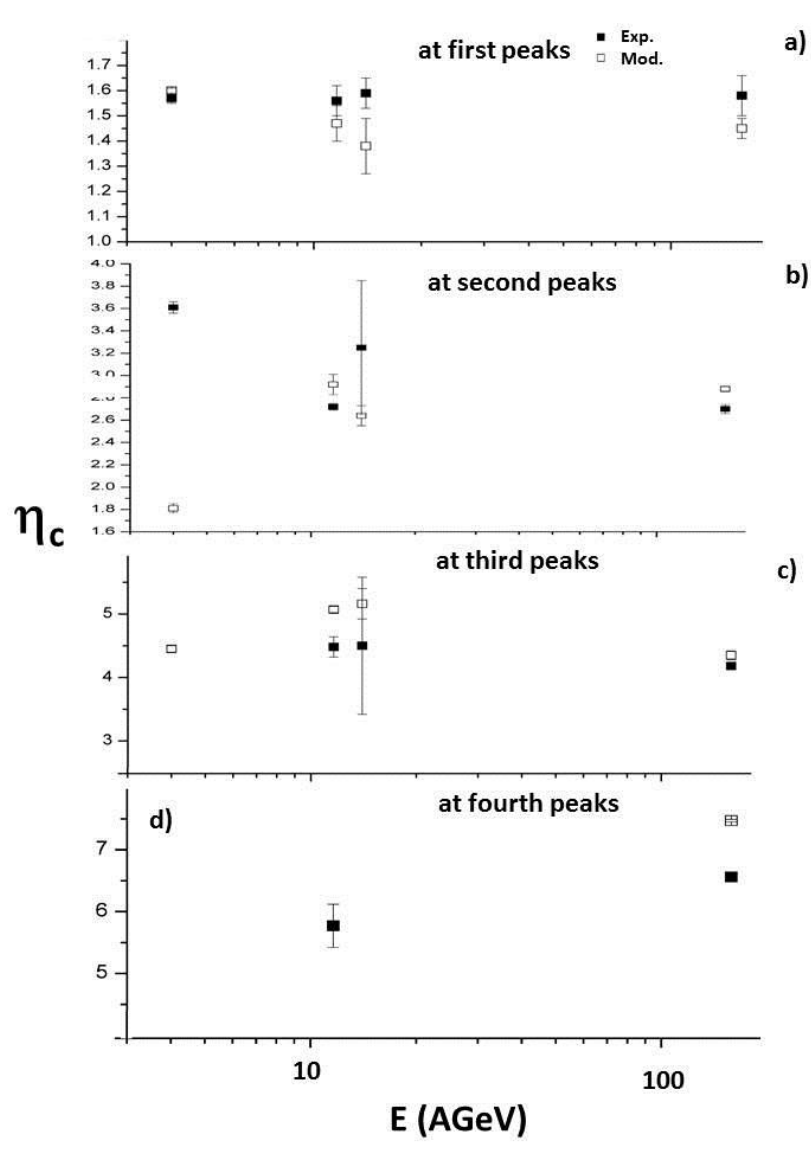

Fig. 4a-d. The values of the pseudorapidity at peaks

5. The first peaks don't depend on the beam energy and could be described completely by the model;

the second peaks depend on the beam energy in the region of $\mathrm{E}<10 \mathrm{AGeV}$ and could not be described completely by the model, in the region of $\mathrm{E}>10 \mathrm{AGeV}$ they don't depend on the energy ;

the third peaks could not be described completely by the model in the region of energy $\mathrm{E}>10 \mathrm{AGeV}$ and don't depend on the energy .

6. The fourth peak observed in case $\mathrm{PbEm}$ (at $158 \mathrm{AGeV}$ )-reactions for experimental events with a number of $\mathrm{g}$ particles $N_{g}=15-20$

7. For the last case the value of the $P 4=6.3 \pm 0.2$, is in the stripping area of $\eta$. But the number of g-particles for this case is $N_{g}=15-20$ which show that these are not stripping particles. So we support that the case may be connected with some special dynamics other than dynamics for 3 previous cases. We believe that in the last case there is some medium effect. To be sure we are going to continue the investigation using other models which include medium effects too. 

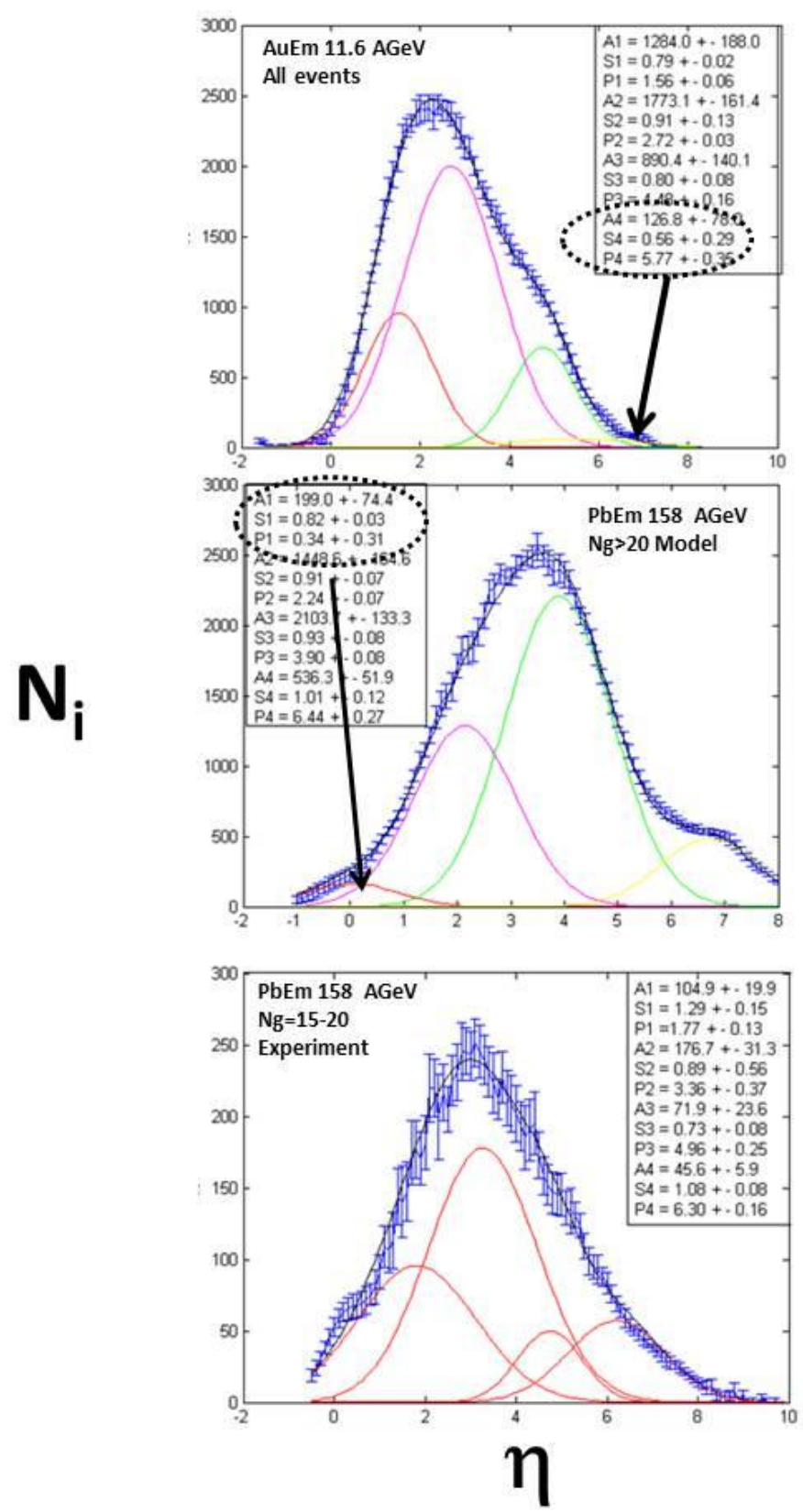

Fig.5.

\section{Acknowledgments.}

Financial support from the HEC Pakistan is cordially acknowledged. 


\section{References}

[1] J.D. Bjorken. Phys.Rev.D, 27, No 1, 140-151, 1983

[2] F. H. Liu, Yu. A. Panebratsev. Nuclear Physics A 641(1998) 379;F. H. Liu and Yu. A. Panebratsev. Physical Review C 59 (1999) 1798; Liu, Fu-Hu. Physical Review C 69 (2004) 067901.

[3] B. Z. Belashev et al. Journal Chine Physics C, 35, N 12 , pp.1095-1099 (2011)

[4] G. Musulmanbekov, Preprint JINR, P2-99-59, Dubna, 1999.

[5] Z. Belashev. Journal of Applied Spectroscopy 68, N 5, pp.838-846 2001

[6] B. Z. Belashev. Int. J Microstructure and Materials Properties 4, No. 3, pp.320-329, 2009

[7] V.P. Dyakonov. Wavelet. From theory to practice. - Solon-,Moscow, pp.217-218, 2002

[8] B. Z. Belashev. Certificate of Rospatent N 2004610196

[9] M. Mitchell. An Introduction to Genetic Algorithms - Cambridge: MIT Press, 1999 - 158 c.-ISBN 0-262-13316-4 (HB), 0-262-63185-7 (PB)

[10] B. R. Hunt, R.L. Lipsman, and J.M. Rosenberg. A Guide to MATLAB, for beginners and experienced users. Cambridge University Press, 2001

[11] B. U. Ameeva et al., Sov.J.Nucl.Phys., 51, 1990, 1047.

[12] M. I. Adamovich et al. (EMU01 - Collaboration), Preprint JINR, E1-92-569, Dubna, 1992.

[13] A. Sh. Gaitinov et al., Proc. of the XVII Meeting of the EMU01 Collaboration, Dubna, Russia, May 18-20, 1999. Dubna, 2000. p.143.

[14] M. I. Adamovich et al., Eur. Phys. J. A., 1999, V.5, p.429 